Department of Social Systems and Management

\author{
Discussion Paper Series
}

No. 1256

Does international knowledge spillover always lead to a positive trickle down?

by

Shiro Kuwahara

March 2010

UNIVERSITY OF TSUKUBA

Tsukuba, Ibaraki 305-8573

JAPAN 


\title{
Does international knowledge spillover always lead to a positive trickle down?*
}

\author{
Shiro Kuwahara ${ }^{\dagger}$ \\ University of Tsukuba
}

March 18, 2010

\begin{abstract}
This paper presents negative effects on economic growth through positive international knowledge spillover, namely, we obtain the possibility of retrogression from human-capital-accumulation-based endogenous growth to non-human-capital-based growth by assuming the phenomenon of international knowledge spillover, effects of population growth on human capital accumulation, and non-unity intertemporal elasticity of substitution on an endogenous growth model with R\&D activities and human capital accumulation. The results show that no growth traps without human capital accumulation in some cases, for example, an economy with a large intertemporal elasticity of substitution and a high population growth rate.
\end{abstract}

Keywords : R\&D-based growth human capital accumulation international knowledge spillover

JEL Classification $\mathrm{O} 11 \cdot \mathrm{O} 34 \cdot \mathrm{O} 41$

\footnotetext{
${ }^{*}$ On this study, I obtain some useful spillover from the joint research with Katsunori Yamada. The research is also financially supported by Japan Society for the Promotion of Science (Wakate B).

†E-mail: kuwahara@sk.tsukuba.ac.jp
} 


\section{Introduction}

Phenomena of tricle down, positive effects on developing neighboring countries from preceding developed countries, is broadly accepted by economists, for example, we can cite Aghion and Bolton (1997) as a work in the literature of endogenous growth theory. However this effects are not always homogeneous, so that, some countries are caught in poverty traps in spite of the neighboring countries' preceding economic development. For example, some empirical works report a polarization of the world economy into the rich and the poor in the modern grobalizing world economy. Therefore, this paper aims to present a theoretical result that there exists a possibility of negative effects of international spillover by using an endogenous growth model with $\mathrm{R} \& \mathrm{D}$ activities through international knowledge spillover and human capital accumulation. Specifically, this paper basically follows the Arnold-type (1998) endogenous growth model, which comprises two growth engines; R\&D activities and human capital accumulation. The former is executed by using the Jones-type innovation function, a function with decreasing returns on existing knowledge and research input. The latter is executed through the Uzawa-Lucas type human capital production function (Uzawa 1965, Lucas 1988).

The Arnold model is developed on the basis of the following discussions. When Romer (1990) developed an early-stage endogenous growth model, he considered a linear relationship between $R \& D$ success and $R \& D$ inputs, which were assumed as human resource and knowledge. This linear relationship captures the non-decreasing returns of knowledge, but leads to theoretical and positive defeats. Although the original model implies that a country with a large population exhibits a higher growth rate, this phenomenon is not supported by positive results. ${ }^{1}$ Furthermore, some positive studies show that knowledge creation functions have decreasing returns properties. This property of the early endogenous growth models is called the scale effect of population. Jones (1995a, 1995b) proposes the incorporating Cobb-Douglas type R\&D function for the Romer model. The input of this function comprises labor (which grows at exogenously given population growth rate) and knowledge. This arrangement can link population growth and endogenous technological change. However, it immediately leads to the next defeat; that is, long-run growth is related to the exogenous population growth rate. Arnold (1998) avoids this defect by assuming that the human resource input to $\mathrm{R} \& \mathrm{D}$ is human capital, which is endogenously accumulated through the UzawaLucas technology.

Our model differs from the basic model of Arnold (1998) with respect to three

\footnotetext{
${ }^{1}$ Theoretically, it cannot contain incessant population growth at its original formation in the steady state.
} 
added factors. The mechanisms of the derived result are as follows: First, we introduce the international knowledge spillover as the same type that is extended in the Arnold model, that is, the world knowledge stock is the sum of the knowledge of the world's countries. Moreover, we assume that the home country is small; therefore, the contribution of accumulation of the home country's knowledge to the dynamics of world knowledge is negligible. Second, our model explicitly captures the effects of population growth on human capital accumulation. With regard to the Jones-type R\&D technology, the positive growth of input factors is necessary for a long-run steady growth path. In Arnold's study (1998), noneducational investment cannot be an equilibrium of a steady state, whereas the population growth effects in our model facilitate the analysis of the steady state with non-educational investment. Third, we assume that there is non-unity intertemporal elasticity of substitution in our model. As a result, the human capital accumulation rate and GDP growth rate are determined by simultaneously, and not separately ${ }^{2}$. Further, the increase in knowledge spillover changes the GDP and human capital accumulation rates. Under some parameter conditions with low intertemporal elasticity of substitution, the growth regime shifts from a positive educational investment regime to a no-educational investment regime. In some cases, for example, in countries with sufficiently high population growth, such an increment decreases the GDP growth rate.

The rest of this paper is organized as follows. The model is constructed in Sec-

\footnotetext{
${ }^{2}$ A simple explanation of separately and simultaneously is provided as follows. We suppose a typical setup of the Uzawa-Lucas model, then the optimal conditions are written as

$$
\begin{aligned}
g_{w}^{*} & =g_{Y}^{*}-g_{H}^{*}=r-b, \\
\theta g_{C}^{*} & =\theta g_{Y}^{*}=r-\rho,
\end{aligned}
$$
}

where $g_{Z}^{*}, w, Y, H, r, b, \theta, C$, and $\rho$ respectively denote growth rate of variable $Z$ in the steady states, wage rate of human capital, output, human capital stock, interest rate, efficiency of education, constant relative risk averse parameter, consumption, and subjective discount rate. Therefore, a steady state is given by

$$
g_{H}^{*}=b-\rho+(1-\theta) g_{Y}^{*}
$$

The equilibrium values of $g_{Y}$ and $g_{H}$ are determined by combining this equation and another relationship between $g_{Y}$ and $g_{H}$ that is derived from final goods production.

The equation $(*)$ shows that in the case of log-linear utility function (namely $\theta=1$ ), $g_{H}$ is determined by the difference of $b$ and $\rho$ and not by $g_{Y}$, and in the case of non-log-linear utility function, it alternatively yields one condition on the relationship between $g_{Y}$ and $g_{H}$. Alternatively, in the simple Uzawa-Lucas model, the condition $g_{H}^{*}=g_{Y}^{*}$ is also derived in the steady state from the structure of the final goods production. In this case, the relationship between $g_{Y}$ and $g_{H}$ is also simply determined as $g_{Y}^{*}=g_{H}^{*}=(1 / \theta)(b-\rho)$ under the condition $\theta \neq 1$. Thus, in the case with $\theta \neq 1$ and $g_{Y}^{*} \neq g_{H}^{*}$, the determination of growth rates is no longer simple, and therefore, we would find some new implications. 
tion 2. Steady states are derived in Section 3. Some growth patterns are analyzed in Section 4. Finally, the conclusion is presented in Section 5.

\section{The Model}

This paper adopts a Romer-type (1990) production structure. In the present analysis, there are three sectors, namely, final goods, intermediate goods, and R\&D, and the following three factors: final goods input to intermediate goods production, human capital employed by final goods production and R\&D input, and knowledge. Knowledge is used in R\&D activities as free input. Following Arnold (1999)'s extension, we introduce international knowledge spillover in the process. Specifically, knowledge input in the R\&D function is not national knowledge, but world knowledge; that is, we assume an open economy with international knowledge accumulation. For simplicity, we omit any international transaction of goods or immigration. Thus, all economies are linked with the world economy only by knowledge. An economy can use the entire world knowledge stock, and the knowledge created by that economy accumulates as a part of the world knowledge stock. Furthermore, we assume that the country in our analyses is sufficiently small; therefore, a country's contribution to the world knowledge stock is also sufficiently small.

This arrangement implies the following two points. First, since we are concerned the dynamics of developing countries, we assume the case of a small country for knowledge accumulation. Second, we are also interested in the role of knowledge spillover; hence, other international factors are have been omitted for simplification.

\subsection{Production}

Final goods can be used for consumption and the production of intermediate goods. They are produced by employing labor, human capital, and a cluster of intermediate goods. ${ }^{3}$ In this paper, human capital can be used for final goods production, investment to create new human capital, and R\&D activities. The aggregate human capital is represented by $H$; the human capitals that are used for final goods production, R\&D activities, and acquisition of human capital stock, such as education, are depicted by $H_{Y}, H_{A}$, and $H_{H}$, respectively. Since all of the above are uses of human capital, the market-clearing condition for human capital imposes $H=H_{Y}+H_{H}+H_{A}$. Intermediate goods are used only in the final goods production process and are supplied monopolistically; this is permitted by

\footnotetext{
${ }^{3}$ In this economy, the scale of the cluster, that is, the cluster of intermediate goods (the variety of the cluster indexed by $A$ ), can be regarded as knowledge.
} 
a patent, which is provided because the firm creates the intermediate goods. One unit of the intermediate good is assumed to be produced by $\eta$ units of the final good, which is used as the numéraire.

The production function of the final goods sector is specified as

$$
Y=H_{Y}^{1-\alpha} \int_{0}^{A} x(j)^{\alpha} d j, \quad 0<\alpha<1,
$$

where $Y, A$, and $X$ are respectively denoted as the final goods product, the number of variety, and intermediate goods input, which is defined by

$$
X:=\int_{0}^{A(t)} x(j)^{\alpha} d j
$$

where $x(j)$ denotes $j$ 's intermediate goods input. ${ }^{4}$ The profit of an intermediate firm with index $j$ is given by

$$
\pi(j)=p(j) x_{t}(p(j))-\eta x_{t}(p(j))
$$

where $p(j)$ is the price of $j$ 's sector intermediate goods.

The final goods sector is assumed to be perfectly competitive; therefore, firms operate under the equating marginal cost and factor price, as shown below:

$$
\frac{\partial Y}{\partial H_{Y}}=w, \quad \text { and } \quad \frac{\partial Y}{\partial x(j)}=p(j)
$$

where $w$ and $p(j)$ denote the wage rate of human capital and the price of $j$ 's sector intermediate goods, respectively.

The intermediate goods are assumed to be monopolized; that is, the firms set the price of these goods for profit maximization. By solving the optimization problem of the intermediate goods firm given in (2), with the optimal conditions in the final goods sector (3), the following conditions are obtained:

$$
x(j)=\left[\frac{\alpha^{2}}{\eta}\right]^{\frac{1}{1-\alpha}} H_{Y}, \quad p(j)=\frac{\eta}{\alpha}, \quad \pi(j)=\left(\frac{1}{\alpha}-1\right) \eta x_{i} .
$$

From (1), $x(j)$ in (4) and the definition of $X$, we obtain

$$
Y=\left[\frac{\alpha^{2}}{\eta}\right]^{\frac{\alpha}{1-\alpha}} A H_{Y}
$$

\footnotetext{
${ }^{4}$ Time index is omitted to ease the burden of notations until we consider dynamics in the latter part of the study.
} 
Since the final goods are consumed as either intermediate goods or consumption goods, (4) and (5) yield

$$
C=Y-\int_{0}^{A} x(j) d j=\left[1-\frac{\alpha^{2}}{\eta}\right] Y(t) .
$$

To ensure a positive consumption, we assume $\alpha^{2}<\eta$.

The profit of the intermediate goods firm can be obtained as

$$
\pi=\alpha(1-\alpha) \frac{Y}{A}
$$

\subsection{R\&D Activities and International Spillover}

$\mathrm{R} \& \mathrm{D}$ is established as a new variety-creating process, and the term of patents of new variety is assumed to be permanent. To eliminate the scale effects, we assume Jones technology, so that the creation of a new variety exhibits diminishing returns to scale. Furthermore, we assume that the stock of knowledge is the world knowledge stock, which is given for this (small) country. Thus, the knowledge function is explanined as follows.

Assumption A We assumed the following R\&D function:

$$
\dot{A}=B \mathfrak{A}^{\chi} H_{A}^{\phi}, \quad \phi \in(0,1), \quad \chi \in[0,1),
$$

where $\dot{Z} \equiv \frac{d Z}{d t}$ is the time derivative of the variable $Z$, and $\mathfrak{A}$ is the world knowledge stock. We assume $\mathfrak{A} \equiv \sum_{\kappa \in S} A_{\kappa}$, where $S$ denotes the set of all countries in the world. Thus, the $i$ th country's knowledge stock $A$ affects the dynamics of $\mathfrak{A}$; however, since we assume that the analyzed country is small, the contribution of the dynamics of the country to the world knowledge stock $\mathfrak{A}$ is negligible.

Free entry into $R \& D$ equates the aggregate cost and profit; this provides the following equations:

$$
v \dot{A}=w H_{A},
$$

therefore,

$$
v=\frac{H_{A}^{1-\phi}}{B \mathfrak{A} \chi} w,
$$

where the value of $R \& D$ equals the present value of perpetual monopoly profits, $v \equiv \int_{0}^{\infty} e^{-\int_{0}^{t} r(s) d s} \pi(t) d t$. The perfect mobility of human capital between final goods production and R\&D sectors equates the wage rates of human capital between these two sectors. We denote this common wage rate as $w$. 
The term of a patent for a newly created variety is assumed to be permanent. Hence, the value of $\mathrm{R} \& \mathrm{D}$ (denoted as $v$ ) can be designated as the present value of perpetual monopoly profits: $v(t) \equiv \int_{t}^{\infty} e^{-\int_{t}^{\tau} r(s) d s} \pi^{M}(\tau) d \tau$. Differentiating this equation with respect to time yields the well-known no-arbitrage condition, which is given as

$$
r(t) v(t)=\pi(t)+\dot{v}(t)
$$

\subsection{Dynamical Optimization of the Household and Human Cap- ital Accumulation}

In the model, the population is denoted as $N$, and it grows at a positive constant exogenous rate $n>0$ (specifically, $\dot{N}(t)=n N(t)$ ). The scale of the representative household is normalized to a unit of the population. The population growth dilutes the representative household's asset holding. The asset holding of the household includes financial assets, that is, equity issued by firms that lead innovation in new intermediate goods, and human assets, which involve human capital accumulated by conscious educational investment for the selection between education and work.

Our study follows the Arnold (1999) model, wherein one knife-edge assumption is eliminated. That is, the Romer-type linear $\mathrm{R} \& \mathrm{D}$ function is replaced by the Cobb-Douglas-type non-linear one. However, Arnold (1999) introduced another knife-edge condition, namely, the log linear utility function. We loosen this linearity and show that the non-log-linear utility function yields the main results of the model.

Assumption $\mathbf{U}$ The utility of the representative agent is given by

$$
\int_{0}^{\infty} \frac{c(t)^{1-\theta}-1}{1-\theta} e^{-\rho t} d t, \quad \theta>\frac{\phi}{1+\phi}(\equiv \underline{\theta})
$$

where $c, \rho$, and $\theta$ represent the per capita consumption, subjective discount rate, and the inverse of the elasticity of intertemporal substitution, respectively. Although we loosen the log-linearity assumption, we still have to impose the domain of the inverse of the elasticity of intertemporal substitution, $\theta \geq \underline{\theta}$. This assumption implies that the intertemporal substitution is not vety high, and simplifies our analysis in the latter part.

The budget constraint in the per capita form is given by,

$$
\dot{a}(t)=r(t) a(t)+w(t)\left(u_{Y}(t)+u_{A}(t)\right) h(t)-c(t)-n a(t),
$$


where $a, u_{Y}, u_{A}$, and $h$ represent the per capita asset holding, allocation rate of human capital to final goods production, allocation rate of human capital to $R \& D$ activities, and the per capita human capital, respectively. It should be noted that $u_{Y}=H_{Y} / H$ and $u_{A}=H_{A} / H$ hold under the assumption of the representative agent.

We assume that the human capital accumulation basically follows the UzawaLucas manner. The human capital is accumulated by human capital investment, and the increment is linear for the investment. Furthermore, since our model explicitly contains population growth, we can clearly capture the effects of population growth in our human capital dynamics. It is assumed that population growth affects two directions of accumulation. First, the representative household's human capital holdings are attenuated by population growth in a usual manner, such as (physical) capital holding in the usual Ramsey model with population growth. Second, we introduce new and mortal factors. We assume that each agent enters the human capital market with a constant endowment. We assume that human capital is also depreciated by factors such as aging or mortality; therefore, we assume that it linearly depends on the population growth rate. Thus, we make the following assumption:

Assumption H Thus, the accumulation of the per capita human capital is shown as follows:

$\dot{h}(t)=b\left(1-u_{Y}(t)-u_{A}(t)\right) h(t)-n h(t)+\delta n h(t), \quad b>0, \quad 0<\delta<\frac{1}{1+\phi}(\equiv \underline{\delta})$,

where $b$ denotes the efficiency of education, and $\delta$ captures a factor of the dynamics of population. The assumption $0<\delta<\bar{\delta}$ implies that human capital accumulation is promoted by exogenous population growth, but its effects are not substantial. The latter part shows that the results of the growth rate for this assumption are consistent with those reported by positive studies.

Aggregating $h$ in the whole economy, we have the following equation:

$$
\dot{H}(t)=b H_{H}(t)+\delta n H(t),
$$

where $H$ and $H_{H}$ are the aggregated stock of human capital and the educational input of human capital respectively.

From the objective function and constraints, the optimal conditions are obtained as follows: With regard to consumption, the usual Keynes-Ramsey rule is given as

$$
\theta \frac{\dot{c}(t)}{c(t)}=\theta\left(\frac{\dot{Y}(t)}{Y(t)}-n\right)=r(t)-\rho-n,
$$

where we use (6) and $C=c N$. 
From the optimal condition of human capital allocation, we obtain

$$
\lambda(t) w(t) \geq \mu(t) b, \quad \text { with equality whenever } \quad u_{H}(t)>0 .
$$

where $\lambda w$, and $\mu$ are denoted as the shadow price of capital, wage rate of human capital, and shadow price of human capital, respectively.

The dynamic equation for $\mu$ is expressed as follows:

$$
\rho-\frac{\dot{\mu}(t)}{\mu(t)}=\frac{\lambda(t) w(t)}{\mu(t)}-b\left(1-u_{Y}(t)-u_{A}(t)\right)-(1-\delta) n
$$

If equality does not exist in condition (16), there is a lack of human capital investment (that is, $u_{H}=0$ ), and the conditions are summarized as follows:

$$
\begin{aligned}
b & <\tilde{\mu}(t) \\
\frac{\dot{\tilde{\mu}}(t)}{\tilde{\mu}(t)} & =\frac{\dot{\lambda}(t)}{\lambda(t)}+\frac{\dot{w}(t)}{w(t)}-\{\rho-\tilde{\mu}(t)+(1-\delta) n\},
\end{aligned}
$$

where $\tilde{\mu} \equiv \frac{\lambda w}{\mu}$.

Finally, the transversality conditions (TVC) are given as follows:

$$
\lim _{t \rightarrow \infty} e^{-\rho t} \lambda(t) a(t)=0, \quad \text { and } \quad \lim _{t \rightarrow \infty} e^{-\rho t} \mu(t) h(t)=0 .
$$

\section{Dynamics and Steady State}

We investigate the dynamics of the economy. We denote the growth rate of the variable $z$ by $g_{z}$ (namel $g_{z} \equiv \frac{\dot{z}}{z}$ ), and value $z$ in the steady growth path (SGP) by $z^{*}$. We also derive the steady states of the model. Our paper contains two growth engine in the model, knowledge accumulation (R\&D) and human capital accumulation. Therefore, we observe four types of steady states: the Arnold regime (positive R\&D and positive human capital accumulation) and the Jones regime (positive R\&D and no human capital accumulation). (5) and (6) imply

$$
g_{Y}(t)=g_{C}(t)=g_{H_{Y}}(t)+g_{A}(t) .
$$

By using (15) and (21), the growth rates are explained as follows.

$$
\theta g_{c}(t)=\theta\left(g_{Y}(t)-n\right)=\theta\left(g_{H_{Y}}(t)+g_{A}(t)-n\right)=r(t)-\rho-n .
$$

If $u_{H}>0$, then $u_{A}+u_{Y}<1$; we can eliminate $\mu$ and obtain the optimal condition as a dynamical equation about $w$ as follows:

$$
g_{w}(t)=r(t)-b-\delta n .
$$


Time differentiating (9), and uniting (11), the following dynamics are obtained:

$$
g_{v}(t)=g_{w}(t)+(1-\phi) g_{H_{A}}(t)-\chi g_{\mathfrak{A}}(t)=r(t)-\frac{\pi(t)}{v(t)} .
$$

We can show that the steady state of the system is saddle stable (see Appendix A.1). Thus, in the main text, we confine our concern on the steady state analysis.

\subsection{Two Types of Long-run Steady Growth Paths}

In this section, we limit our attention to the case of SGP, and derive some features of the model. In the present model, we have two SGPs: one path for the internal solution case about human capital accumulation (with $H_{H}>0$ ) and the other path for the corner solution case about the same (with $H_{H}=0$ ).

First of all, the human capital of each allocation must grow at constant rate(s); $H_{H}>0$ yields $g_{H_{A}}^{*}=g_{H_{Y}}^{*}=g_{H_{H}}^{*}=g_{H}^{*}$ and $h_{H}=0$ yields $g_{H_{A}}^{*}=g_{H_{Y}}^{*}=g_{H}^{*}=\delta n$ and $g_{H_{H}}^{*}=0$. From (8) and $g_{H_{A}}=g_{H}$, the following condition is necessary for the steady growth equilibrium,

$$
g_{A}^{*}-\chi g_{\mathfrak{A}}^{*}=\phi g_{H_{A}}^{*} .
$$

Notice here that Jones technology under international knowledge spillover affects $g_{A}$ through the efficiency parameters $(\chi$ and $\phi)$ and international knowledge growth rate, but by the level parameter $a$.

(21) implies that, in the steady state, the following equation holds:

$$
g^{*}=g_{H}^{*}+g_{A}^{*} .
$$

By combining (25), and (26), we obtain the condition relating the growth rate of human capital to the growth rate of GDP as

$$
g^{*}=(1+\phi) g_{H}^{*}+\chi g_{\mathfrak{A}}^{*} .
$$

\subsection{The Arnold Regime (A-Regime)}

Since the Arnold (1998) model contains human capital accumulation derived by the Uzawa-Lucas educational function and R\&D activities driven by Jones technology, we term the growth regime with these factors as the Arnold regime (Aregime).

When $h_{H}>0, w=(1-\alpha) \frac{Y}{H_{Y}}$, and (23) imply that the following equation holds in the steady state:

$$
g^{*^{A}}-g_{H}^{*^{A}}=r^{*^{A}}-b-\delta n,
$$


where $*^{A}$ indexes the steady state in A regime.

(5) and $w=(1-\alpha) \frac{Y}{H_{Y}}$ imply $g_{w}=g_{A}$, and (22) implies $r^{*^{A}}=\theta\left(g_{Y}^{*^{A}}-n\right)-$ $\rho-n$. Substituting these two properties into (28) yields

$$
g_{H}^{*^{A}}=\frac{1}{\Upsilon}\left[-(1-\theta) n+(b+\delta n-\rho)+(1-\theta) \chi g_{\mathfrak{A}}^{*}\right] .
$$

where $\Upsilon \equiv 1-(1-\theta)(1+\phi)$. From (27) and (29), the growth rates of GDP and per capita GDP are given as

$$
\begin{aligned}
& g^{*^{A}}=\frac{1+\phi}{\Upsilon}\left[-(1-\theta) n+(b+\delta n-\rho)+\frac{\chi}{1+\phi} g_{\mathfrak{A}}^{*}\right], \\
& g_{y}^{*^{A}}=\frac{1}{\Upsilon}\left[-n+(1+\phi)(b+\delta n-\rho)+\chi g_{\mathfrak{A}}^{*}\right],
\end{aligned}
$$

where $y \equiv Y / N$. It should be noted that this can be equilibria if the conditions for A-regime are satisfied.

We can observe from (30) to (29) that each growth rate of the A-regime is a linear combination of the factor of the population growth rate $n$, the factor of contribution of the usual Uzawa-Lucas type human capital accumulation $b-\rho$, and the additive term $\delta n$, which is an added factor in the Uzawa-Lucas type human capital accumulation in Assumption $H$. It should be noted that $\Upsilon>0$ under the assumption $\theta>\underline{\theta}$, and that $1 / \Upsilon$ functions as a multiplier of growth factors such as $n$ and $b-\rho$.

\subsection{The Jones Regime (J-Regime)}

The above discussion solves steady state by assuming that $h_{H}^{*}>0$, which implies $g_{H}>\delta n$. However, this condition is not always satisfied. We term the growth regime only with $R \& D$ activities driven by Jones technology as the Jones regime (J-regime).

Therefore, if condition $g_{H}^{*}>\delta n$ is lacking, then the economy is stuck in the equilibrium $h_{H}=0$; therefore, uniting these properties and (28), we obtain the steady state human capital accumulation rate and knowledge growth rate in this regime as

$$
g_{H}^{*^{J}}=\delta n, \quad \text { and } \quad g_{A}^{*^{J}}=\phi \delta n+\chi g_{\mathfrak{A}}^{*} .
$$

Therefore, combining (27) and (31) yields the GDP growth rate on the economy caught in a no-education trap as Under the condition $H_{H}=0$, we obtain the aggregate and per capita GDP growth rates in this regime as

$$
\begin{aligned}
& g^{*^{J}}=(1+\phi) \delta n+\chi g_{\mathfrak{A}}^{*}, \\
& g_{y}^{*^{J}}=(1+\phi) \delta n+\chi g_{\mathfrak{A}}^{*}-n,
\end{aligned}
$$


(32) shows that the per capita GDP growth rate exhibits a semi-endogenous growth property: the growth rate is pinned down to the population growth rate and exogenous world knowledge growth rate.

\section{Regime Determination}

We derive regime determination in this section. As for the study of the relationship between population growth and the per capita GDP growth rate, the empirical studies of Kellry (1988), Kelley and Schumidt (1995), and Ahituv (2001) report (weak) negative correlation between the per capita income growth and population growth. Thus, we here assume that $\partial g_{y}^{*} / \partial n<0$. This assumption and the steadystate per capita GDP growth rate $g_{y}^{*}$ yield the following condition:

$$
v \equiv 1-(1+\phi) \delta>0, \quad \text { equivalently } \quad \delta<\frac{1}{1+\phi}(=\underline{\delta}) .
$$

Therefore, under the assumed arrangement of not very large effects of population growth on human capital accumulation, our model satisfies the property $\partial g_{y}^{*} / \partial n<$ 0 .

Since A-regime is a case of inner solution about human capital investment, we have the feasibility condition on human capital accumulation. (14) yields $g_{H}^{*} \in$ $[\delta n, b+\delta n]$. Thus, the lower bound condition $g_{H}>\delta n$ provides

$$
g_{H}^{*^{A}}>\delta n \Leftrightarrow b>\rho+(\theta-1)\left(\chi g_{\mathfrak{A}}^{*}-v n\right),
$$

and the upper bound condition $g_{H}<b+\delta n$ provides

$$
\begin{aligned}
g_{H}^{*^{+}}<b+\delta n \Leftrightarrow b\left\{\begin{array}{c}
< \\
>
\end{array}\right\} \frac{1}{1+\phi}\left(\frac{\rho}{1-\theta}+v n-\chi g_{\mathfrak{A}}^{*}\right), \\
\qquad \text { for }\left\{\begin{array}{c}
1>\theta>\underline{\theta} \\
\theta>1
\end{array}\right.
\end{aligned}
$$

It can be easily checked that the transversality conditions corresponds with (34). Thus, for $g_{H}^{*^{A}}$ to be the steady state value, both (33) and (34) must hold.

For the J-regime to be in a steady state, (18) and (19) provide the following conditions;

$$
\begin{aligned}
& b<\tilde{\mu}^{*^{J}} \\
& g_{\lambda^{*}}+g_{w^{*}}-\left\{\rho-\tilde{\mu}^{*^{J}}-(1-\delta) n\right\}=0,
\end{aligned}
$$


where $*^{J}$ represents the index of the value in the J-regime. Substituting $g_{Y}^{*^{J}}$ and $g_{H}^{*^{J}}$ derived in (32)-(31), and $g_{\lambda}=-\theta\left(g_{Y}-n\right)$ and $g_{w}=g_{Y}-g_{H}$ into the above conditions, we have

$$
\text { J-regime } \Leftrightarrow \quad b<\rho+(\theta-1)\left(\chi g_{\mathfrak{A}}^{*}-v n\right)
$$

Thus, the conditions (33) and (37) imply that the equation

$$
b=\rho+(\theta-1)\left(\chi g_{\mathfrak{A}}^{*}-v n\right)\left(\equiv L B\left(\theta ; g_{\mathfrak{A}}^{*}\right)\right)
$$

divides the parameter domain into A-regime and J-regime. The name of "LB" stems from the lower bound, that is, this line makes the lower bound of positive human capital investment.

This domain is depicted in Figure 1, which contains two cases: that of higher growth rate of world knowledge $\left.g_{\mathfrak{A}}^{* *}\right)$ and that of the lower one $g_{\mathfrak{A}}^{*}\left(<g_{\mathfrak{A}}^{* *}\right)$. The main dividing line is the LB-Line, which is shifted by the change of $g_{\mathfrak{A}}^{*}$. From Figure 1, we can determine the domain where the higher $g_{\mathfrak{A}}^{*}$ makes a country from A-regime to J-regime. Thus, we have the following Lemma:

Lemma I If $\theta$ is larger than 1, that is, the intertemporal elasticity of substitution is small, the increase in the growth rate of world knowledge spillover results in some countries in A-regime falling into J-regime.

We assume the knowledge growth rate increase from $g_{\mathfrak{A}}^{*}$ to $g_{\mathfrak{A}}^{* *}\left(>g_{A}^{*}\right)$, and this makes a country shift from A-Regime to J-Regime. Therefore, the steady state changes from A-regime with the world knowledge growth rate $g_{\mathfrak{A}}^{*}$ to J-regime with the world knowledge growth rate $g_{\mathfrak{A}}^{* *}$. We respectively denote the per capita GDP growth rates of these two steady states as $g_{y}^{* A}\left(g_{\mathfrak{A}}^{*}\right)$ and $g_{y}^{* J}\left(g_{\mathfrak{A}}^{* *}\right)$. Then, we check the change of per capita growth rate between $g_{y}^{* A}\left(g_{\mathfrak{A}}^{*}\right)$ and $g_{y}^{* J}\left(g_{\mathfrak{A}}^{* *}\right)$. It should be noted that this situation is $\theta>1$ from the Lemma I, therefore, $\Upsilon>1$ from the definition.

The difference of $g_{y}^{A *}\left(g_{\mathfrak{A}}^{*}\right)$ and $g_{y}^{* J}\left(g_{\mathfrak{A}}^{* *}\right)$ is shown as follows:

$$
\begin{aligned}
\Delta_{g_{y}} & \equiv g_{y}^{* J}\left(g_{\mathfrak{A}}^{* *}\right)-g_{y}^{* A}\left(g_{\mathfrak{A}}^{*}\right) \\
& =\frac{1}{\Upsilon}[\underbrace{\chi\left(\Upsilon g_{\mathfrak{A}}^{* *}-g_{\mathfrak{A}}^{*}\right)}_{+} \underbrace{-(\theta-1)(1+\phi) v n}_{-}-\underbrace{(1+\phi)(b-\rho)}_{ \pm}] .
\end{aligned}
$$

Therefore, for example, if the population growth rate is very high, the regime change from $\mathrm{A}$ to $\mathrm{J}$ leads to a decrease in the growth rate of the country. 
Lemma II If $\Delta_{g_{y}}<0$, for example, smaller power of world knowledge stock in the $R \& D$ function $(\chi)$, higher population growth rate, and education efficiency is higher than the subjective discount rate ${ }^{5}$, the increase of the growth rate of international knowledge stock generates the regime switch from A-regime to Jregime, and causes the decrease of a county's per capita GDP growth rate.

From the above two Lemmas, the proposition of this study is stated as follows:

Proposition If a country has a large intertemporal elasticity of substitution and a high population growth rate, an increase in the world knowledge growth rate might cause the county to fall into low-growth traps without educational investment.

\section{Conclusion}

This study develops an endogenous growth model that comprises variety expansion, human capital accumulation, and international spillover. The non-unity CRRA parameter (Assumption U), human capital accumulation affected by population growth (Assumption $\mathrm{H}$ ), and R\&D function with international knowledge spillover (Assumption A) relate the determination of the growth phase with the population growth rate and world knowledge growth rate, and in some domains, the increase in the international knowledge spillover might have a negative effect on countries' growth rate.

\section{A Dynamical System and Steady States}

The analysis is simplified by using variables that are constant in steady states, we define new variables $u_{Y} \equiv H_{Y} / H$ and $u_{A} \equiv H_{A} / H$. By using these notations, it can be written as $H_{H}=\left(1-u_{Y}-u_{A}\right) H$.

From the discussions in Section 2, the model has two types of regime: one is the case with education, and the other is that without education. In both the cases, the economies follow the rules of common dynamics (8), (24), (14), and (22). (8) and (14) are respectively noted as

$$
\begin{aligned}
g_{A}(t) & =g_{A}\left(u_{A}(t), \xi(t)\right)=B\left(u_{A}(t) \xi(t)\right)^{\phi}, \\
g_{H}(t) & =g_{H}\left(u_{A}(t), u_{H}(t)\right)=b\left(1-u_{A}(t)-u_{Y}(t)\right)+\delta n,
\end{aligned}
$$

\footnotetext{
${ }^{5}$ It should be noted that this condition $(b-\rho>0)$ is the condition for positive long-run growth in the Uzawa-Lucas model. Furthermore, if this condition is not satisfied, extremely high population growth can lead to the same result.
} 
where $\xi \equiv H /\left(A^{1 / \phi} \mathfrak{A}^{-\chi / \phi}\right)$.

(21) and $w$ in (3) yields

$$
\frac{\dot{w}(t)}{w(t)}=\frac{\dot{Y}(t)}{Y(t)}-\left(\frac{\dot{u}_{Y}(t)}{u_{Y}(t)}+g_{H}(t)\right)=\frac{\dot{A}(t)}{A(t)}
$$

Plugging (7), (10) and (42) into (24) yield

$$
r(t)=\frac{\dot{A}(t)}{A(t)}+(1-\phi)\left[\frac{\dot{u}_{A}(t)}{u_{A}(t)}+g_{H}(t)\right]-\chi g_{\mathfrak{A}}(t)+\alpha \frac{u_{Y}(t)}{u_{A}(t)} g_{A}(t)
$$

Differentiating $\xi$ with respect to time, and substituting (41) and (40) into it yields

$$
\dot{\xi}(t)=\left[g_{H}\left(u_{Y}(t), u_{A}(t)\right)-\frac{1}{\phi} g_{A}\left(u_{A}(t), \xi(t)\right)+\frac{\chi}{\phi} g_{\mathfrak{A}}(t)\right] \xi(t)
$$

\section{A.1 The Case of Arnold Regime}

Adding (23) and (42), this case contains the following optimization condition:

$$
r(t)-b-\delta n=\frac{\dot{w}(t)}{w(t)}=\frac{\dot{A}(t)}{A(t)} .
$$

Eliminating $r$ and $w$ by uniting (43) and (45), we have the dynamics of $u_{A}$ in the internal solution case as

$$
\dot{u}_{A}(t)=\left\{-g_{H}\left(u_{Y}(t), u_{A}(t)\right)+\frac{b+\delta n+\chi g_{\mathfrak{A}}(t)-\alpha \frac{u_{Y}(t)}{u_{A}(t)} g_{A}\left(u_{A}(t), \xi(t)\right)}{1-\phi}\right\} u_{A}(t) .
$$

Eliminating $r$ and $w$ by using (22) and (45), we have

$$
\frac{\dot{u}_{Y}(t)}{u_{Y}(t)}=-g_{H}(t)+b+\delta n-\rho+(1-\theta) \frac{\dot{Y}(t)}{Y(t)} .
$$

Substituting (21) into the above equation, and solving with respect to $\dot{u}_{Y}$, we have the following dynamics of $u_{Y}$ in the internal solution case as

$$
\begin{aligned}
\dot{u}_{Y}(t)= & \left\{\frac{b-\rho}{\theta}+\frac{\delta n}{\theta}\right. \\
& \left.-g_{H}\left(u_{Y}(t), u_{A}(t)\right)+\frac{1-\theta}{\theta} g_{A}\left(\xi(t), u_{A}(t)\right)\right\} u_{Y}(t),
\end{aligned}
$$


From (40) and (41), $g_{A}$ and $g_{H}$ are functions that depend on $u_{A}, u_{Y}$, and $\xi$. Thus, all equations, (44), (46), and (47), are a function with variables $u_{A}, u_{Y}$, and $\xi$, and the dynamics of the system are completely represented by these three dynamics.

The linearized system of $q, \kappa, \xi, u_{Y}$, and $u_{A}$ around a steady state is written as

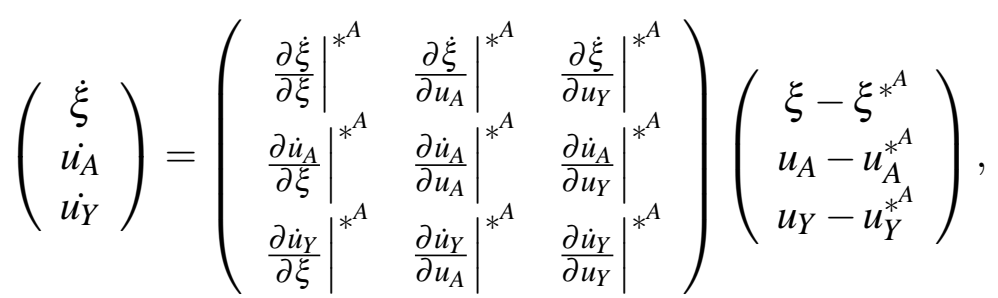

where, by using

$$
\left.\frac{\partial g_{A}}{\partial \xi}\right|^{*^{A}}=\phi \frac{g_{A}^{*^{A}}}{\xi^{* A}},\left.\quad \frac{\partial g_{A}}{\partial u_{A}}\right|^{*^{A}}=\phi \frac{g_{A}^{*^{A}}}{u_{A}^{*^{A}}}, \quad g_{H}^{*^{A}}=\frac{1}{\phi} g_{A}^{*^{A}}-\frac{\chi}{\phi} g_{\mathfrak{A}}^{*},
$$

and $b\left(u_{A}^{*}+u_{Y}^{*}\right)=b+n-g_{H}^{*^{A}}$, we can derive the following:

$$
\begin{aligned}
& \left.\frac{\partial \dot{\xi}}{\partial \xi}\right|^{*^{A}}=-g_{A}^{*^{A}},\left.\quad \frac{\partial \dot{\xi}}{\partial u_{A}}\right|^{*^{A}}=-\left(b+\frac{g_{A}^{*^{A}}}{u_{A}^{*^{A}}}\right) \xi^{*^{A}}, \\
& \left.\frac{\partial \dot{\xi}}{\partial u_{Y}}\right|^{*^{A}}=-b \xi^{*^{A}},\left.\quad \frac{\partial \dot{u}_{A}}{\partial \xi}\right|^{*^{A}}=\Gamma_{1} u_{A}^{*^{A}},\left.\quad \frac{\partial \dot{u}_{A}}{\partial u_{A}}\right|^{*^{A}}=\Gamma_{2} u_{A}^{*^{A}}, \\
& \left.\frac{\partial \dot{u}_{A}}{\partial u_{Y}}\right|^{*^{A}}=\Gamma_{3} u_{A}^{*^{A}},\left.\quad \frac{\partial \dot{u}_{Y}}{\partial \xi}\right|^{*^{A}}=\frac{1-\theta}{\theta} \phi \frac{g_{A}^{*^{A}}}{\xi^{* A}} u_{Y}^{*^{A}}, \\
& \left.\frac{\partial \dot{u}_{Y}}{\partial u_{A}}\right|^{*^{A}}=\left[b+\frac{1-\theta}{\theta} \phi \frac{g_{A}^{*^{A}}}{u_{A}^{*^{A}}}\right] u_{Y}^{*^{A}},\left.\quad \frac{\partial \dot{u}_{Y}}{\partial u_{Y}}\right|^{*^{A}}=b u_{Y}^{*^{A}},
\end{aligned}
$$

where

$$
\Gamma_{1}=-\frac{\alpha \phi}{1-\phi} \frac{u_{Y}^{*^{A}}}{u_{A}^{*^{A}}} \frac{g_{A}^{*^{A}}}{\xi^{*^{A}}}, \quad \Gamma_{2}=b+\alpha \frac{u_{Y}^{*^{A}}}{\left(u_{A}^{*^{A}}\right)^{2}} g_{A}^{*^{A}}, \quad \Gamma_{3}=b-\frac{\alpha}{1-\phi} \frac{g_{A}^{*^{A}}}{u_{A}^{*^{A}}} .
$$

The characteristic equation is assumed to be given as

$$
\Psi(\omega)=-\omega^{3}+\operatorname{Tr}^{*^{A}} \omega^{2}-B{J^{* A}}^{A} \omega+\operatorname{Det}^{*^{A}} .
$$

From (46), we obtain

$$
\alpha \frac{u_{Y}^{*}}{u_{A}^{*}} g_{A}^{*}=-(1-\phi) g_{H}^{*}+b+\delta n+\chi g_{\mathfrak{A}}^{*}
$$


Considering this and (25), we can prove that the trace $T r^{*+}$ is positive as follows:

$$
\begin{aligned}
\operatorname{Tr}^{*^{A}} & =\left.\frac{\partial \dot{\xi}}{\partial \xi}\right|^{*^{A}}+\left.\frac{\partial \dot{u}_{A}}{\partial u_{A}}\right|^{*^{A}}+\left.\frac{\partial \dot{u}_{Y}}{\partial u_{Y}}\right|^{*^{A}} \\
& =\underbrace{b\left(u_{A}^{*}+u_{Y}^{*}\right)}_{>0}+\underbrace{b+\delta n-g_{H}^{*^{A}}}_{>0}>0,
\end{aligned}
$$

where $b+\delta n-g_{H}^{*^{A}}>0$ is obtained from the property that the upper bound of $g_{H}$ is $b+\delta n$.

The determinant $D e t^{* A}$ is derived as follows:

$$
\begin{aligned}
D e t^{*^{A}} & =-g_{A}^{*^{A}} \Gamma_{2} u_{A}^{*^{A}} b u_{Y}^{*^{A}}-\left[b+\frac{g_{A}^{*^{A}}}{u_{A}^{*^{A}}}\right] \xi^{*^{A}} \Gamma_{3} u_{A}^{*^{A}} \frac{1-\theta}{\theta} \phi \frac{g_{A}^{*^{A}}}{*^{* A}} u_{Y}^{*^{A}} \\
& +b \xi^{*^{A}} \Gamma_{1} u_{A}^{*^{A}}\left[b+\frac{1-\theta}{\theta} \phi \frac{g_{A}^{*^{A}}}{u_{A}^{*^{A}}}\right]+g_{A}^{*^{A}} \Gamma_{3} u_{Y}^{*^{A}}\left[b+\frac{1-\theta}{\theta} \phi \frac{g_{A}^{*^{A}}}{u_{A}^{*^{A}}}\right] \\
& +\left[b+\frac{g_{A}^{*^{A}}}{u_{A}^{*^{A}}}\right] \xi^{*^{A}} \Gamma_{1} u_{A}^{*^{A}} b u_{Y}^{*^{A}}+b \xi^{*^{A}} \Gamma_{2} u_{A}^{*^{A}} \frac{1-\theta}{\theta} \phi \frac{g_{A}^{*^{A}}}{*^{* A}} u_{Y}^{*^{A}} \\
& =b u_{A}^{*^{A}} u_{Y}^{*^{A}} g_{A}^{*^{A}}\left[1-\frac{1-\theta}{\theta} \phi\right]\left(\Gamma_{1}-\Gamma_{2}+\Gamma_{3}\right) .
\end{aligned}
$$

From $\Gamma_{1}, \Gamma_{2}$, and $\Gamma_{3}$, it is easily derived as

$$
\Gamma_{1}-\Gamma_{2}+\Gamma_{3}=-\frac{\alpha}{1-\phi} \frac{u_{Y}^{*^{A}}}{u_{A}^{*^{A}}} g_{A}^{*^{A}}\left(\frac{1-\phi}{u_{A}^{*^{A}}}+\frac{1}{u_{Y}^{*^{A}}}\right)-\frac{\phi}{\xi *^{A}} \alpha \frac{u_{Y}^{*^{A}}}{u_{A}^{*^{A}}}<0 .
$$

Uniting $\theta>\frac{\phi}{1+\phi}$, which is equivalent to $1-\frac{1-\theta}{\theta} \phi>0$, and (50), we obtain the result of $\mathrm{Det}^{*^{*}}<0$. From the results of $\operatorname{Tr}^{*^{A}}>0$ and $D e t^{*^{A}}<0$, we can conclude that the system that we are currently studying has saddle stable property under the assumption of $\Upsilon>0$ in the long-run.

\section{A.2 The Case of the Jones Regime}

We are primarily interested in the case of negative trickle-down, so we assume $\theta>1$ in this section.

This regime imposes the following condition:

$$
u_{A}(t)+u_{Y}(t)=1
$$


(51) makes (14) as

$$
\dot{H}(t)=\delta n H(t), \quad \text { namely } \quad g_{H}(t)=\delta n=g_{H}^{*^{J}}
$$

(52) states that the rate of human capital accumulation in this case is constant. The value of corner-solution steady state is denoted by $*^{J}$.

From (40), (51), (52) and the definition of $\xi$, the dynamics of $\xi$ are obtained as

$$
\dot{\xi}(t)=\left[\delta n-\frac{1}{\phi} g_{A}(t)+\frac{\chi}{\phi} g_{\mathfrak{A}}(t)\right] \xi(t)
$$

(15) and (32) give

$$
r=\rho+n+\theta\left\{(1+\phi) \delta n+\chi g_{\mathfrak{A}}-n\right\} .
$$

Substituting (40), (51), (52) and (54) into (43), we have the dynamics of $u_{A}$ as follows:

$$
\dot{u}_{A}(t)=\left\{\frac{\Gamma_{4}+(1+\theta) \chi g_{\mathfrak{A}}(t)-\left(1+\alpha \frac{1-u_{A}(t)}{u_{A}(t)}\right) g_{A}(t)}{1-\phi}\right\} u_{A}(t) .
$$

where $\Gamma_{4} \equiv \rho+n+\{(1+\theta) \phi+\theta-1\} \delta$.

Thus, the system of this case comprises of two dynamics of $\xi$, depicted by (53) and $u_{A}$ depicted by (55).

We can obtain the values in a steady state as follows. Eliminating $g_{\mathfrak{A}}^{*}$ by using (53) and (55), we obtain $u_{A}^{* J}$ as follows:

$$
u_{A}^{*^{J}}=\frac{\alpha\left(\phi \delta n+\chi g_{A}^{*}\right)}{\Gamma_{4}+(1+\theta) \chi g_{\mathfrak{A}}^{*}-(1-\alpha)\left(\phi \delta n+\chi g_{\mathfrak{A}}^{*}\right)} .
$$

If $u_{A}^{* J} \in(0,1)$ is satisfied, $u_{A}^{* J}$ can be an equilibrium, and uniting this $u_{A}^{J *}, g_{A}^{*^{J}}$ derived in (31) and (40) yields $\xi^{* J}$. These $\left(u_{A}^{* J}, \xi^{* J}\right)$ give the steady state. Thus, the steady state of Jones regime is also uniquely given if all feasible conditions are satisfied.

We derive $\dot{\xi}=0$ and $\dot{u_{A}}=0$ loci. (53) and (55) respectively yield $\dot{\xi}=0$ and $u_{A}=0$ locis as

$$
\begin{gathered}
\dot{u}_{A}=0 \text {-loci }: \quad \xi=U\left(u_{A}\right)=\left[\frac{\Gamma_{4}+(1+\theta) \chi g_{\mathfrak{A}}^{*}}{B\left(1+\alpha \frac{1-u_{A}}{u_{A}}\right)}\right]^{\frac{1}{\phi}} u_{A}^{-1}, \\
\dot{\xi}=0 \text {-loci }: \quad \xi=\Xi\left(u_{A}\right)=\left[\frac{\phi \delta n+\chi g_{\mathfrak{A}}^{*}}{B}\right]^{\frac{1}{\phi}} u_{A}^{-1} .
\end{gathered}
$$


It can easily be shown that $U^{\prime}(\cdot)<0, \Xi^{\prime}(\cdot)<0$, and $U(1)>\Xi(1)$. Furthermore, we can derive

$$
\begin{gathered}
\dot{u}_{A}\left\{\begin{array}{c}
> \\
<
\end{array}\right\}=0 \Longleftrightarrow \xi\left\{\begin{array}{c}
< \\
>
\end{array}\right\} U\left(u_{A}\right) . \\
\dot{\xi}\left\{\begin{array}{c}
> \\
<
\end{array}\right\}=0 \Longleftrightarrow \xi\left\{\begin{array}{c}
< \\
>
\end{array}\right\} \Xi\left(u_{A}\right) .
\end{gathered}
$$

From these properties, the existence condition for the steady state and its uniqueness, we have the phase diagram of the J-regime as describe in Figure 2, and we determine find the saddle stability of this regime.

\section{References}

[1] Aghion, P. and P. Bolton, A Theory of Tricle-Down Growth and Development, Review of Economic Studies, 64, (1997)

[2] Ahituv, A., Be fruitful or multiply: On the interplay between fertility and economic development, Journal of Population Economics, 14, 51-71 (2001)

[3] Arnold, L. G., Growth, welfare, and trade in an integrated model of humancapital accumulation and research, Journal of Macroeconomics, 20(1), 81105 (1998)

[4] Jones, C. I., Time series tests of endogenous growth models, Quarterly Journal of Economics, 110, 495-525 (1995a)

[5] Jones, C. I., R\&D-based models of economic growth, Journal of Political Economy, 103, 759-784 (1995b)

[6] Kelley, A., Economic consequence of population change in the third world, Journal of Economic Literature, 26, 1685-1728 (1988)

[7] Kelley, A., Schmidt, R., Aggregate population and economic growth correlations: The role of the components of demographic change, Demography, $32,543-555$ (1995)

[8] Lucas, R. E. Jr., On the mechanism of economic development, Journal of Monetary Economics 22(1), 3-42 (1988)

[9] Romer, P. M., Endogenous Technological Change, Journal of Political Economy, 98(5), S71-S102 (1990)

[10] Uzawa, H., An aggregative model of optimal technical change, International Economic Review, 6, 18-31 (1965) 


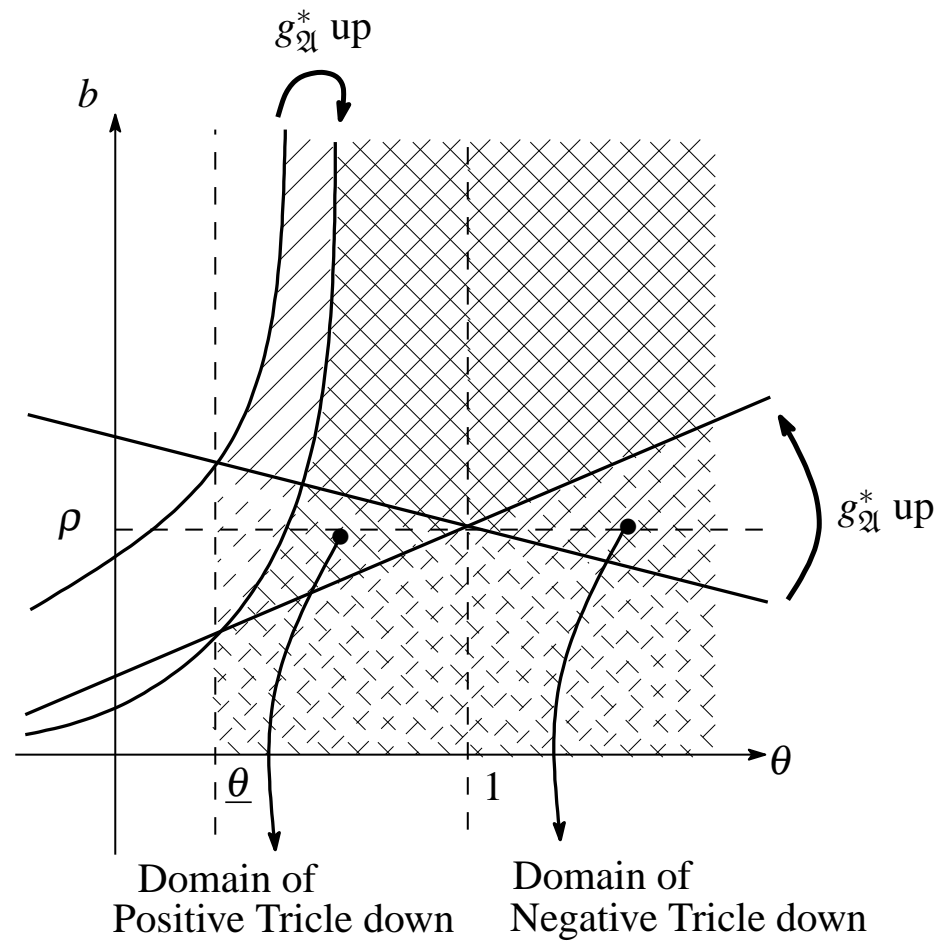

\begin{tabular}{|c|c|c|}
\hline & For $g_{\mathfrak{A}}^{*}$ & For $g_{\mathfrak{A}}^{* *}\left(>g_{\mathfrak{A}}^{*}\right)$ \\
\hline A-Regime & & \\
\hline J-Regime & 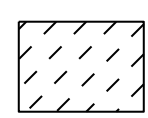 & 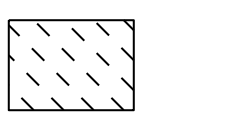 \\
\hline
\end{tabular}

Figure 1: Growth regimes 


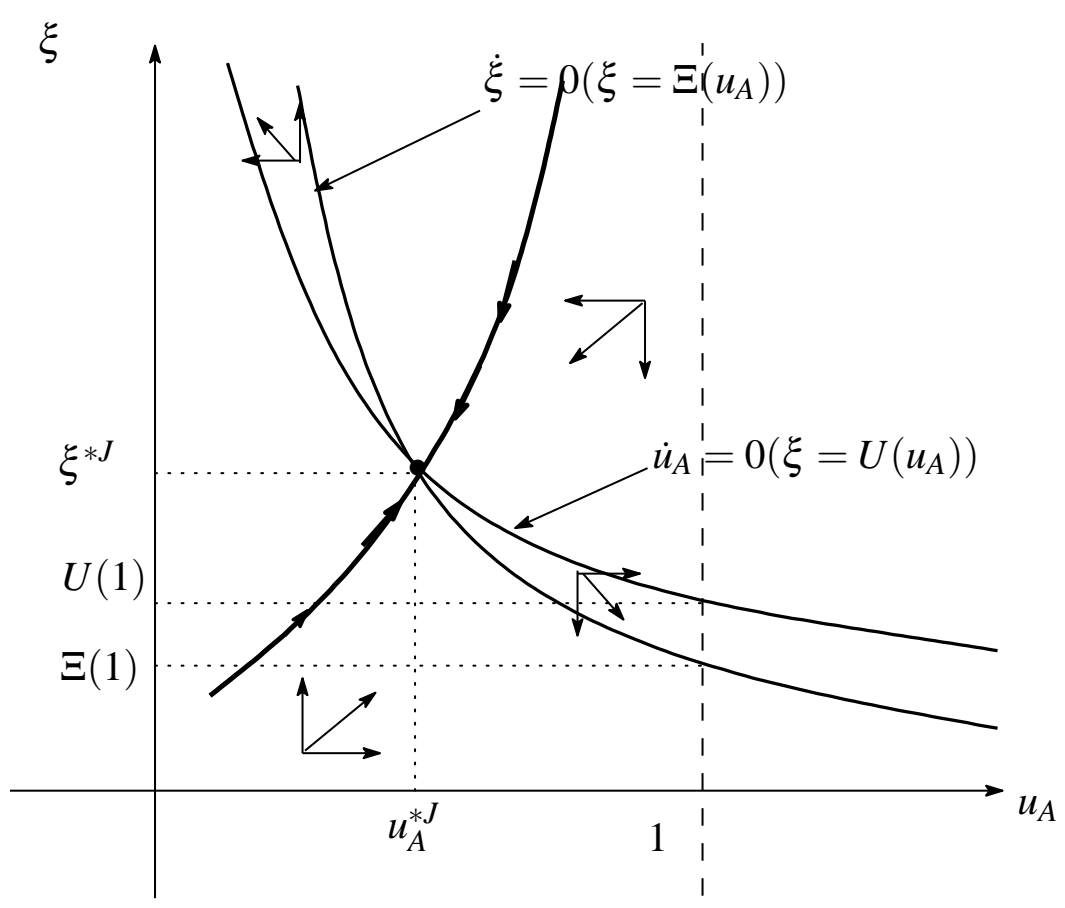

Figure 2: Phase diagram of J-regime 